\title{
«NOTAS EN TORNO A LA POSICIÓN DEL ADJETIVO Y A LA DOBLE ADJETIVACIÓN EN LAS LENGUAS INDOEUROPEAS»
}

\author{
Mariano Benavente \\ Universidad de Jaén
}

I. Es posible que el título de este trabajo resulte en exceso largo y a la par pretencioso. Nos preocupa, empero, desde hace tiempo el escaso espacio prestado, en los manuales más al uso y en otras obras de más pretendida enjundia, a la posición adjetival y a la doble adjetivación, tanto en el ámbito de las lenguas clásicas como en el de otras lenguas indoeuropeas, muertas o vivas. En efecto, por lo que hace al primer punto, si prescindimos de los obligados párrafos dedicados a las posiciones atributivas o predicativas del ajetivo' 0 a tfmidas matizaciones de orden estilístico ${ }^{2}$; hallamos un silencio de tumba egipcia, que nos resulta poco justificable. En lo tocante al segundo punto, la omisión y desinterés parecen ser mayores todavía.

Pero volviendo la mirada a los textos, según vieja costumbre nuestra, del griego micénico, griego clásico, latín, antiguo indio, antiguo irlandés, gótico y, dando un gran salto en el tiempo, a los de distintas lenguas cultas de la actual

1 Cf. A. Debrunner en E. Schwyzer, Gr. Gramm., II, München, 3.' ed. 1996-II, pp. 25-27 y 179-181; A. Meillet, Introduction d l'étude.... reimpr. Alabama, 1964, pp. 360-362.

3 Id id, pp. 703-704; A. Emout-F. Thomas, Syntaxe latine, Paris, 2.' ed. 1953, pp. 162-163; 0. Riemann, Syntaxe latine, Paris, 7.' ed. 1942, pp. 13-23; C.W. Conrad, From Epic to Lyric. A Study in the history of traditional word-order in Greek Poetry..., New York-London, 1990, Pp. 45-71. 
Europa; se nos reafirma la idea de que en el indoeuropeo el adjetivo, fuera determinativo o calificativo, debió de poder ir, con notoria libertad, antepuesto o pospuesto al sustantivo acompañado. Si a ello añadimos algunos ejemplos de doble adjetivación en el védico, griego micénico, griego y latín clásico, reforzados por los casos de anfítesis adjetival ${ }^{3}$ que hallamos en lenguas modernas como el español ${ }^{4}$, gallego, griego actual, francés e italiano, amén de otros casos de doble adjetivación, antepuesta o pospuesta al nombre, en idiomas como el inglés y algunos de los más arriba nombrados; será fácil concluir, sin mucha audacia pero con buena lógica, que este doble adjetivo con un solo sustantivo puede retrotraerse asimismo al indoeuropeo.

De otro lado, y como helenista en particular, me siento un tanto defraudado ante el ominoso silencio que envuelve y cubre los frecuentes y bellos ejemplos de anfítesis adjetival que hallamos en poetas tan importantes como Homero, Hesíodo, Píndaro, Baqunlides y los tres grandes trágicos. Para no incurrir en reprobable desorden expositivo, pasamos a examinar a continuación, y con el pertinente orden, uno y otro aspecto.

\section{LA POSICIÓN DEL ADJETIVO EN LAS LENGUAS INDOEUROPEAS:}

Con respecto a la colocación del adjetivo, hallamos una cierta rigidez e inflexibilidad en idiomas de la moderna Europa que han fijado, por el uso y de modo un tanto monótono, esta posición dicha. Pero conviene recordar que no hay regla sin excepción y que, incluso en unos pocos ejemplos del inglés (cf. «The Theatre Royal», «from time inmemorial», «the Poet Laureate», etc.) $)^{5}$ aparece el adjetivo pospuesto al sustantivo (cosa que suele ser silenciada o ignorada, por cierto, en muchos manuales que pasan por buenos). Por ello no es de extrañar que otras lenguas europeas de hoy admitan, de modo más o menos alternante o excepcional, esta misma y doble posición del adjetivo, como es el caso del ruso ${ }^{6}$. Si nos volvemos a las lenguas muertas como, verbi-

${ }^{3}$ Entendemos que hay anfitesis cuando un adjetivo, determinativo o calificativo, precede al nombre y otro le sigue.

4 Estamos preparando al presente, con la valiosa cooperación de I. Ahumada, excelente hispanista y companero en la Universidad de Jaén, una extensa monografia sobre el tema, en la que se recogen ejemplos que abarcan, prácticamente, todos los distintos períodos de la literatura española.

'Cf. C.E. Eckersley, A Concise English Grammar, London, 2.' ed. 1951, p. 17 y S. Ulimann, Semántica, introducción a la ciencia del significado, trad. esp., Madrid, 1970, p. 192.

- Según que aparezca la llamada kestructura largan o kestructura breve». Cf. R. Jakobson, Slavic Languages, New York, 1955², s.v. «East Slavic, Russian». 
gracia, el antiguo irlandés (cf. «cobled móm = «fiesta grande», frente a «in tres fer» = «el tercer hombre» ${ }^{7}$ ); latín clásico (cf. «aurea domus», frente a «nauis oneraria»); griego micknico (cf. «ko-ri-si-jo ra-pte-re = Korinsioi rapteres» = «zurcidores corintios» (?), frente a «ko-wo me-zo-e $=$ korwoi mezoes» $=$ «mu-

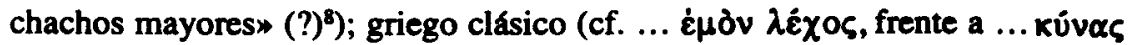

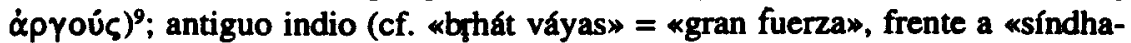
vas mayobhúvas» $=$ <rios beneficiosos $)^{10}$; gótico (cf. $*$ faheid mikila $=$ kalegria grande», frente a «us managaim hairtam» = «de muchos corazones»" rece queda clara esta doble posibilidad de anteponer o posponer el adjetivo, determinativo o calificativo, al sustantivo al que acompañe. De ahf que, al existir una y otra colocación, el adjetivo antepuesto resulte, a veces, más subjetivo y enfático, o bien el énfasis recaiga sobre la construcción menos frecuente ${ }^{12}$. Se trata, en todo caso, de distribuciones de índole estilística en cada lengua en particular. Es evidente, por ejemplo, que en idiomas en los que se ha regularizado la anteposición del adjetivo, como ocurre en inglés, alemán, danés y sueco, verbigracia, la ocasional y excepcional posposición del mismo proporcionará una construcción más expresiva y, quizá, más literaria ${ }^{13}$.

\section{LA DOBLE ADJETIVACIÓN CON UN SOLO SUSTANTIVO:}

Dejando aparte el hecho bien conocido de que pueden yuxtaponerse varios adjetivos referidos a un único nombre o a un sujeto no explícito (cf., respectivamente, védico «sugús asat suhiranyás svásvas...» = «es de esperar que sea rico en vacas, rico en oro, rico en caballos.... ${ }^{14}$ y, pasándonos a nuestro Juan Ramón, «Platero es pequeño, peludo, suave...»), aquí estudiamos los casos en que un solo nombre lleva, antepuestos o pospuestos, dos adjetivos, 0 , en más equilibrada construcción, cuando surge la anfítesis adjetival, esto es, cuando un adjetivo precede al sustantivo y otro le sigue. Se trata, en suma, de tres

7 Cf. J. Pokorny, Antiguo irlandes, Madrid, 1952, pp. 14 y 40 y 45.

- Cf. E. Vilborg, A tentative Grammar of Mycenaean Greek, Goteborg. 1960, p. 138.

- Cf. Hom., II, vv. 31 y 50, respectivamente.

10 Cf. F.R. Adrados, Védico y sánscrito clasico, Madrid, 1953, p. 123.

1 Cf. A. Tovar, Lengua gotica, Madrid, 1946, pp. $43-44$.

12 Cf. Ullmann, ob cit., pp. 155-156, 192 y 291; M. Criado de Val, Fisonomfa del idioma español, Madrid, 1954, pp.30-31.

${ }^{13}$ Cf. Ullmann, ob. cit., p. 192; R. Lenz, La oración y sus partes, Madrid, 1925, pp. 178-179; P. Chantraine, La stylistique grecque, Paris, 1951, pp. 9-10; J.S. Lasso de la Vega, Sintaxis griega, Madrid, 1967, pp. 90-96.

14 Cf. F. R. Adrados, ob. cit., p. 123. 
posibilidades de las que la tercera, la anfítesis, testimoniada ya en védico y en griego micénico, como a continuación veremos, aparece con frecuencia en la poesía griega antigua. También la hallamos en los textos latinos clásicos, el Romancero español ${ }^{15}$, nuestros escritores de los siglos de oro, el Neoclásico... Sin interrupción en las letras hispanas, alcanza las cotas de ilustres poetas y prosistas del siglo $\mathrm{XX}^{16}$. Y tenemos interesantes ejemplos de anfitesis adjetival en otros eximios escritores de otras literaturas, sirvan los nombres, como botón de muestra, de Kostas Kavafis ${ }^{17}$ y Celso Emilio Ferreiro ${ }^{18}$. Mas antes de hacer hincapie en la anfítesis, que, por ser más bella construcción, ha alcanzado, sin duda, mayor éxito en los ámbitos literarios, veamos también algunos ejemplos de las otras dos posibles construcciones referidas, no sin advertir antes que no nos vamos a ceñir, tan sólo, a ejemplos con adjetivos «sensu strictow, sino también a todos aquellos en que los participios o cualquier otra voz adjetivada intervienen:

A) Doble adjetivación antepuesta al sustantivo:

Abunda en ambas lenguas clásicas y está ya documentada en algunos versos fórmula de Homero:

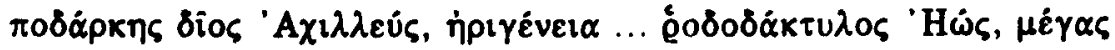

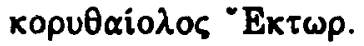

También aparece en versos virgilianos como «...magna comitante caterva» (En. II, 40 y 371); «... lenta... superaddita uitis» (Egl. III, 38); «strata... sua... poma» (Egl. VIII, 54).

Tambien la encontramos, como anticipábamos, en el inglés literario: «...strong white teeth...» (Kipling, The Jungle Book, cap. I, Ifnea 11), «... naked brawn baby...* (Id id id, línea 113); «... little naked cubs...» (Id id id, línea 158).

B) Doble adjetivación pospuesta al sustantivo:

De esta construcción hay ejemplos en antiguo indio:

«...gavấ iva subhré mãtára rihané...» ${ }^{19}$. Asimismo los hay en latín:

is Cf., por ejemplo, «blanco rostro encendidow, «loco rey mancebow, «débil mujer sola», «primer bozo negro», «mi jardín florecido*, «sus jomadas contadas», «aquel postigo viejo», «mal caballero probadow, en R. Menéndez Pidal, Flor Nueva de Romances Viejos, Madrid, 1976, pp. 45 $46,56,74,164$ y 168.

16 Abunda en la poesía de A. Machado y F. Garcfá Lorca, en J. Ramón Jiménez (Platero y yo) y en J. Ortega y Gasset (Castilla y sus castillos), por ejemplo. VEanse ejs. en J. Lens-M. Benavente, Pindaro, Píticas, introd., trad. y notas (en prensa), cf. nota 100 a Pftica IV.

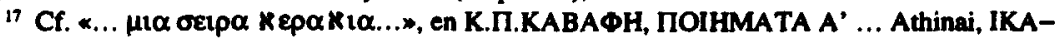
noर, 1963, P. 97.

1" Cf. «lonxanos ros camifantesw, «dondos ácios áticosw, vv. 21 y 7, respectivamente, de «Pranto por Carlés Ribaw, en p. 46 de Longa noite de pedra, Barcelona, 1967.

19 Cf. F.R. Adrados, ob. cit., p. 126. 
«...Androgeos...credens inscius... ${ }^{20}$; griego clásico y griego moderno: Cf.,

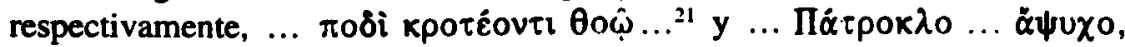

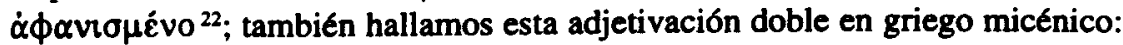
«to-pe-za e-re-pa-te-ja po-ro-e-ke = torpeza alephanteia proekhes» = kuna mesa marfileña, saliente... $»^{23}$. Y está, por supuesto, documentada en múltiples lenguas cultas modernas.

C) La doble adjetivación en anfítesis:

Tenemos algunos interesantes ejemplos en védico:

«...yáh saptáraśmir vrṣabhás túvismản...» = «el que indomable ${ }^{24}$ toro fuerte...25; «bhadrás ásvãs harítas Súryasya...» = «los brillantes caballos overos del Sol (Surya).... ${ }^{26}$.

Y también encontramos la anfítesis, como ya hemos dicho, en griego micénico:

«... ku-te-se-jo e-ka-ma-pi e-re-pa-te-jo-qe» = «kuteseois ekhamappi elephanteoisque» $=$ (literalmente) $*$...con ebanales adomos marfileños $»$, esto es, «con adomos de ébano y marfil» ${ }^{27}$.

En la poesía griega antigua:

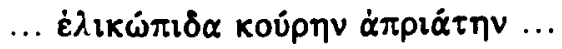

$\mathbf{A}$

98-99

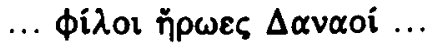

B

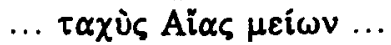

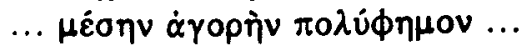

B

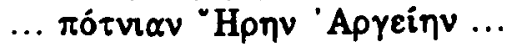

B

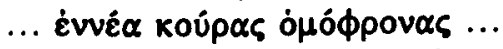

Hes., Th.

60

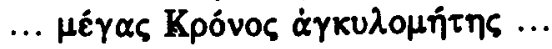

Hes., Th.

Hes., Th. $168,473,495$

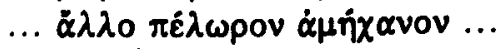

Hes., Th.

295

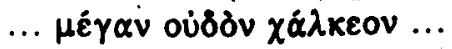

Hes., Th.

749-750

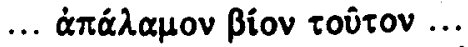

Pi., O. I

59

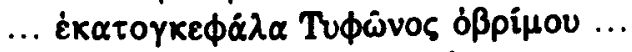

Pi., O. IV

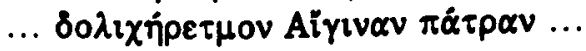

Pi., O. VIII

20 Cf. Virg., En. II 371-372.

2 Cf. Pi., Pac. V(= fr. 52) 17 Snell-Machler.

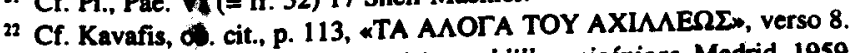

${ }^{23}$ Cf. M. Ferníndez-Galiano, Diecisiete tablillas micénicas, Madrid, 1959, pp. 193-196.

24 Literalmente, «que necesita siete riendasw, cf. Adrados, ob. cit., p. 193, s.v. ks a p tá r a smix.

25 Cf. Adrados, misma ob. cit., p. 125.

26 Id id id, p. 123.

27 Cf. E. Vilborg, ob. cit., p. 139. 


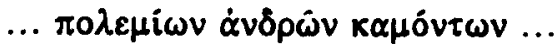

Pi., P. I

80

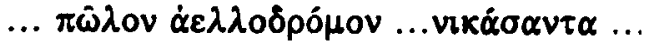

B., V

$39-40$

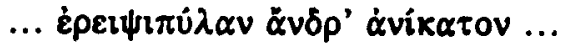

B., $\mathbf{V}$

$56-57$

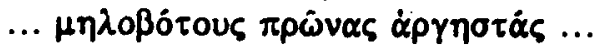

B., $\mathrm{V}$

$65-66$

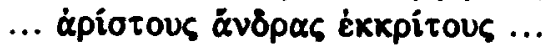

A., Th.

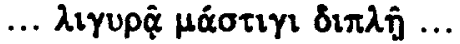

S., Aj.

242

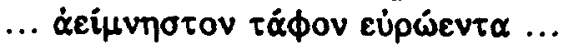

S., Aj.

1166-67

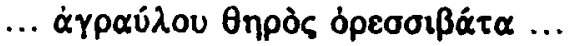

S., Ant.

349-350

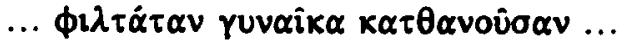

E., Alc.

231-232

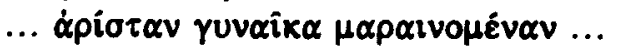

E., Alc.

235-236

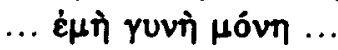

E., Alc.,

329-330.

En la literatura latina:

Primus... Laocoon ardens... Virg., En. II 40-41.

...tua carmina digna... Virg., Egl. VIII 10

...recentem Romanam pacem... T. Liv., XXI, 2

...scripta legione noua... T. Liv., XXI, 25

...Numidas equites quingentos... T. Liv., XXI, 29

Como puede comprenderse, hay muchos casos de doble adjetivación, en ambas lenguas clásicas, en que los adjetivos aparecen dispuestos según las construcciones estudiadas en los apartados A) y B), esto es, dos adjetivos ante el sustantivo o detrás de el, pero que, a efectos de traducción, son vertidos en anfítesis, sea por influencia de los textos en que ésta aparece en verdad, sea porque, como hemos apuntado más arriba, resulta más equilibrada y bella la construcción en anfítesis, o sea por casual elección del traductor. Pero el hecho cierto es que, en aquellas lenguas que no están amarradas al duro banco de la inflexible y obligada posición adjetival, como ocurre, en general, en las lenguas latinas, la anfítesis adjetival pulula triunfante y su uso llega a ser, en ocasiones, no sólo abusivo sino también algo indigesto ${ }^{28}$. Su proliferación, en efecto, puede resultar un punto fatigosa en autores cuyos textos son de gran valía y belleza, pero que quizá hagan excesivo empleo de esta doble adjetivación ${ }^{29}$.

28 En cierto libro poético, publicado en 1941, en Valladolid, aparecen hasta ocho ejemplos de anfitesis, en el espacio de treinta y siete versos...

29 Juan Ramón Jiménez, verbigracia, hace uso de esta doble adjetivación, en Platero y yo, hasta noventa y siete veces, lo cual equivale a decir que hallamos casi un ejemplo por capítulo; Federico García Lórca la emplea en unos ciento cincuenta pasajes, a lo largo de las 1196 páginas de sus versos (cf. A. del Hoyo, Federico García Lorca, Obras completas, tomo I. Verso, Madrid, 1987). 
Por supuesto, al hacer estas últimas afirmaciones somos conscientes de que nos estamos adentrando en un peligroso lodazal de sujetivismo (en el que a veces se hunden conspicuos tratadistas y tratados, por cierto ${ }^{30}$ ). Y por ello aquí ponemos punto final a estas breves notas.

30 Albin Lesky, por ejemplo, en su excelente manual de literatura griega, dice, en página 778 de la versión espaniola (Madrid, Gredos, 1968), refiriéndose a Herodas, wal que sólo con ciertas reservas puede llamársele poetaw. Nosotros nos preguntamos, con cierta inquietud en nuestro magnánimo corazón, si, en caso de ser el primer hombre que publicó los mimos de Herodas un seffor llamado, verbigracia, Schneider, Schroeder o Strauss, en vez de serlo Mr. Kenyon; se hubiera mostrado el Profesor Lesky tan despectivo en su juicio sobre el autor de los mimos. Casos como tste hay muchos y nosotros sentimos ante ellos auténtica vergüenza ajena. 